\title{
Mathematical modeling for chemistry-related applications
}

\author{
J. Vigo-Aguiar
}

Received: 7 November 2012 / Accepted: 8 November 2012 / Published online: 28 November 2012 (C) Springer Science+Business Media New York 2012

We are pleased to offer readers of Journal of Mathematical Chemistry this special issue consisting of four mathematical models selected for their implications in several branches of chemistry. This collection constitutes a state of art in the subject.

The article of Pilar Castro et al. discusses a mathematical model for representing the thermal flow created by a point heat source and transmitted through a medium made up of borehole drill cuttings. This model is important because it is the basis of an experiment which makes it possible to determine underground thermal properties using a method which is simple, inexpensive, and sufficiently accurate for the aims of this line of research. Knowledge of these properties is a key element when designing shallow geothermal systems.

The paper of Ranilla et al. was selected because there has been a great interest in studying the effect of primordial chemistry on the Cosmic Microwave Background (CMB) fluctuations. Line absorption, photoionozation and photodissociation leave their imprint on the CMB power spectrum. From the point of view of chemistry, determining with great accuracy the $\mathrm{CMB}$ fluctuations is very important to put constraints on the proportions of different molecules in the Early Universe. In this work, a tool based on parallel computing and high performance computing applied to detection and flux estimation in CMB maps is presented. This tool improves the accuracy and performance of existing ones.

The main contribution of the paper "note on the generalized Rayleigh equation: limit cycles and stability" written by Miguel A. López and Raquel Martínez is to analyze the dynamics (limit cycles and stability) of a generalization of the Rayleigh equation. Recall that there is a continuous dynamical system given by an oscillator and it models a batch distillation process where a fixed quantity of feed is charged

\footnotetext{
J. Vigo-Aguiar $(\bowtie)$

Department of Applied Mathematics, University of Salamanca, Salamanca, Spain e-mail: jvigo@usal.es
} 
to the distillation column and separation of volatile products is done by supplying heat through the still or reboiler. As the boiling point of liquid is reached, a portion of liquid starts to vaporize to the top of the column and directed to the condenser, the vapor leaving the column is always at equilibrium with the liquid in the bottom of the column, thus a material balance equation is produced by the Rayleigh approach.

Finally, I am glad to present a trilogy of papers by Professor Metin Demiralp's group. The nonlinearity is perhaps the most undesired thing in applied mathematics and people try to find some ways to avoid that. The Probabilistic Evolution Approach (PEA) is one of the most recently developed tool to bypass nonlinearity in ODEs. To this end, Demiralp proposed a new method to get solutions to ODEs and related problems like initial or boundary value problems. The method was based on a basis of function expansion of the descriptive functions. However, instead of very well-known multivariate Taylor series, the Kronecker power series, which are not unique unless certain impositions like equipartitions of identical terms are employed for the variant expansion coefficients, are used as the basic expression. This produces a linear but infinite set of homogeneous ODEs with a constant infinite matrix which facilitates the analysis. Although the fundamentals of this theory are now quite well established, there are still new advances in details and new openings; some are announced through conference proceedings and journal papers.

Even though the basic inspiration has been taken from the expectation value dynamics of the quantum mechanics, this has been a rather comprehensive presentation of how PEA can be formulated for the evaluation of quantum dynamical expectation values. The first paper of this trilogy is devoted to this task. There the expectation values for a system vector with operator elements, together with its Kronecker powers have been expressed as the solution of an infinite ODE with an infinite coefficient matrix. There it has been shown that the cases where the infinite coefficient matrix is triangular faciliate especially the spectral analyses. Beyond that multinomiality, where that matrix has few adjacent nonzero upper diagonals together with the main diagonal, this brings further simplifications. The conicality, where the triangular coefficient matrix has main and one upper diagonal having nonzero elements only, enables us to use the first order recursive structures. This has been presented in the first paper of this trilogy. The convergence for the coefficients of the Kronecker power series has also been analyzed and certain norm including results have been reported. This is an important development for the mathematical chemists. This approach does not require the evaluation of the wave function and therefore the solution of the Schrödinger equation. The initial form of the wave function suffices for our purposes.

The second paper of the trilogy collects the information related to PEA for managing singularities and the structural simplifications in the coefficient matrix (Evolution Matrix). These issues are also essential and in fact have not been considered as secondary items. However, it has been preferred to present them in a separate part, for the facilitation of the reading. The first and second papers of the trilogy have been devoted to the quantum mechanical issues and, when it is considered together with the PEA for ODE systems which may come not from quantum mechanics, the universality of the Probabilistic Evolution Approach becomes really understandable. The nonlinearity is removed at the expense of infinite dimensionality. However, all investigations 
imply that the appropriately defined truncation approximants work quite well in many applications.

The evidence of the PEA universality is not limited to only ODEs and quantum expectations. It has also been shown that an equivalent application of the approach to the Liouville equation is successful and again an infinite set of homogeneous ODEs with a constant infinite coefficient matrix appears. ODEs are generally for our daily life applications and describe the things in human dimensionality. The quantum expectation values, despite the fact that they can be renormalized to human dimensionalities, are for the micro world, the realm of atoms, molecules etc. On the other hand, the Liouville equation, and therefore the density and partition functions, describe the unmanageably populated systems like systems including several Avogadro number particles, that is, the systems which can be managed statistically only. The third paper of the trilogy emphasizes the fact that PEA can also work for these extensions.

As a final statement, this trilogy has been organized to illustrate the capabilities and amenabilities of PEA for various areas in theoretical chemistry. However, they should not be considered as the elixir of life or philosopher's stone. This trilogy is just a good and promising beginning for inviting researchers to further development and enrichment of this theory.

We do believe that this collection of articles can be useful and informative on the new perspectives and trends in computational chemistry and we hope that the reader can enjoy reading them, hoping that this can also serve as inspiration for new outcomes. The origin of these articles is the series of conferences on computational mathematics and applications. The 12th International Conference on Computational and Mathematical Methods in Science and Engineering (CMMSE 2012) was held in La Manga (Spain), from 2 to 6 July 2012. A special session on Computer science and mathematics for chemistry-related applications was included in this event. The present issue is a selection of papers in these sessions.

Finally, as guest editors, we would like to express our gratitude to all the authors and referees for their help in contributing to this special volume [1-10] .

\section{References}

1. J. Vigo-Aguiar, S. Gray, I. Hamilton (eds.), Computer science and mathematics for chemistry-related applications. J. Math. Chem. 48 (1) (2010). 50(2), 379-380 (2012)

2. J. Vigo-Aguiar, J.L.G. Guirao (eds.), Advances in computational and mathematical chemistry. Special issue CMMSE-2010. 50(2), 311-312 (2012)

3. P. Alonso, P. Ian Hamilton, J. Vigo-Aguiar (eds.), Mathematical and computational methods with applications in chemistry and physics. Special issue CMMSE-2009. J. Math. Chem. 48(1) (2010)

4. J. Vigo-Aguiar, E.J. Brändas (eds.), Mathematical and computational tools in theoretical chemistry. Special issue CMMSE-2008. J. Math. Chem. 48(1) (2010)

5. J. Vigo-Aguiar, B.A. Wade (eds.), Special issue CMMSE-2005. Dedicated to Prof. Erkki J. Brändas. J. Math. Chem. 40(1) (2006)

6. J. Vigo-Aguiar, Special issue on computational and mathematical methods in science and engineering (CMMSE-2004). J. Comput. Appl. Math. 192(1) (2006)

7. J. Vigo-Aguiar, B.A. Wade, Recent advances in computational and applied mathematics in science and engineering. Int. J. Comput. Math. 85(3-4), 307 (2008)

8. J. Vigo-Aguiar, G. Vanden Berghe, Advances in computational and mathematical methods in science and engineering. J. Comput. Appl. Math. 235(7), 1745 (2011) 
9. T.E. Simos, J. Vigo-Aguiar, Special issue-selected papers from the conference on computational and mathematical methods for science and engineering (CMMSE-2002). J. Comput. Appl. Math. 158(1) (2003)

10. T.E. Simos, J. Vigo-Aguiar, Special issue for the international conference of computational methods in scienceand engineering, 2003 (ICCMSE 2003). Math. Comput. Model. 42(7-8) (2005) 